\title{
Prospective Comparative Study of Preoperative Expectations and Postoperative Outcomes in Anatomic and Reverse Shoulder Arthroplasty
}

\author{
Cassandra Lawrence $^{1}$ Mark Lazarus ${ }^{2}$ Joseph Abboud ${ }^{2}$ Gerald Williams ${ }^{2}$ Surena Namdari² \\ ${ }^{1}$ Department of Orthopaedic Surgery, Sidney Kimmel Medical College at \\ Thomas Jefferson University, Philadelphia, Pennsylvania, United States \\ Address for correspondence Surena Namdari, MD, MSc, Division of \\ Shoulder \& Elbow Surgery, Rothman Institute-Thomas Jefferson \\ 2 Department of Orthopaedic Surgery, Rothman Institute-Thomas \\ University, 925 Chestnut Street, 5th Floor, Philadelphia, PA 19107, \\ Jefferson University, Philadelphia, Pennsylvania, United States \\ United States (e-mail: surena.namdari@rothmaninstitute.com).
}

Joints 2019;7:159-164.

\begin{abstract}
Keywords

- expectations

- reverse arthroplasty

- total shoulder arthroplasty

- exercise

Background Compared with anatomic total shoulder arthroplasty (aTSA), reverse total shoulder arthroplasty (RTSA) is associated with lower preoperative and postoperative outcome scores and range of motion. It is unknown whether patients' preoperative expectations of surgery are lower in RTSA compared with aTSA. The purpose of this study was to assess preoperative patient expectations and postoperative outcomes in aTSA and RTSA.

Methods A consecutive series of patients undergoing primary aTSA for diagnosis of osteoarthritis or primary RTSA for diagnosis of rotator cuff tear arthropathy were studied prospectively. Expectations were evaluated using the validated Hospital for Special Surgery's Shoulder Surgery Expectations Survey. Baseline demographics, comorbidities, and social factors were collected. Baseline and 2 years postoperative American Shoulder and Elbow Surgeons (ASES) score, visual analog scale pain, Single Assessment Number Evaluation (SANE), and patient satisfaction were obtained.

Results There were 128 patients (64 aTSA and 64 RTSA). There was no significant difference in total preoperative expectations score between groups. On multivariate linear regression analysis, aTSA $(p=0.024)$ and younger age $(p=0.018)$ were associated with higher expectations for improved ability to exercise. Changes in preoperative to postoperative ASES $(p=0.004)$ and SANE $(p=0.001)$ scores were higher in the aTSA group. Total preoperative expectations score was not correlated with postoperative functional outcomes or satisfaction in either group. In the aTSA group, expectations for participation in exercise were positively correlated with changes in preoperative to postoperative ASES score $(p=0.01)$ and SANE score $(p=0.01)$.

Conclusion Though patients undergoing primary aTSA demonstrated greater improvement in functional outcome than those undergoing primary RTSA, both groups reported similar aggregate preoperative expectations. Those undergoing aTSA had higher expectations for return to exercise which was positively correlated with postoperative functional outcomes.

Level of Evidence Level II, prospective cohort study.
\end{abstract}

received

May 21, 2019 accepted after revision

April 18, 2021

published online

June 18, 2021
DOI https://doi.org/ $10.1055 / \mathrm{s}-0041-1730982$. ISSN 2282-4324.

\footnotetext{
(C) 2021. The Author(s).

This is an open access article published by Thieme under the terms of the Creative Commons Attribution-NonDerivative-NonCommercial-License, permitting copying and reproduction so long as the original work is given appropriate credit. Contents may not be used for commercial purposes, or adapted, remixed, transformed or built upon. (https://creativecommons.org/ licenses/by-nc-nd/4.0/) Georg Thieme Verlag KG, Rüdigerstraße 14, 70469 Stuttgart, Germany
} 


\section{Introduction}

Previous studies have validated the quantification of patientreported expectations of surgery. ${ }^{1}$ Recent investigations in the orthopaedic literature have reported an association between expectations of surgery and patient-perceived reporting of preoperative pain and function. ${ }^{1-4}$ In general, patients' expectations of shoulder surgery vary by demographics, diagnosis, and functional status. ${ }^{1}$ It has been shown that patients undergoing anatomic total shoulder arthroplasty (aTSA) with worse shoulder function have higher expectations for improved self-care and daily activities. ${ }^{4}$

While the most common indication for aTSA is osteoarthritis and the most common indication for reverse total shoulder arthroplasty (RTSA) is rotator cuff tear arthropathy, patients undergoing both procedures typically complain of disabling pain and loss of motion. Though both procedures provide improvements in pain relief and restoration of function, the outcomes can vary significantly. In a comparison of aTSA and RTSA surgical outcomes, aTSA was associated with higher preoperative and postoperative outcome scores and greater postoperative range of motion..$^{5}$ More limited final range of motion, higher hospital readmission rates, and higher complication rates can be expected with RTSA compared with aTSA. ${ }^{5-7}$ Greater preoperative expectations of aTSA have been associated with better outcomes. ${ }^{8}$

It is unknown whether patients who are undergoing aTSA or RTSA have differences in preoperative expectations. Knowledge of these variables may provide insight into the modifiable and nonmodifiable factors that could contribute to the shaping of patient expectations. The primary goal of this study was to compare preoperative expectations of patients undergoing aTSA for a diagnosis of osteoarthritis and RTSA for a diagnosis of cuff tear arthropathy and to determine whether there is association with postoperative functional results. Second, we sought to identify variables that were associated with expectations. We hypothesized that patients undergoing aTSA would have higher expectations and better postoperative functional results than patients undergoing RTSA.

\section{Methods}

After obtaining Institutional Board Review approval, patients who underwent primary aTSA for a diagnosis of osteoarthritis and RTSA for the diagnosis of cuff tear arthropathy at a single institution were prospectively studied. Patients undergoing revision surgery, those with a history of prior shoulder arthroplasty, and those with other diagnoses, such as avascular necrosis, proximal humerus fracture, or inflammatory arthropathy, were excluded. Patients from the practices of five fellowship trained shoulder surgeons were included in the study, and standardized consent forms and patient information materials were utilized.

The data collected from the preoperative evaluation included patient demographics, body mass index (BMI), selfassessed comorbidities, marital status, preoperative measurements of active forward elevation, previous nonarthro- plasty shoulder surgeries, and use of depression or anxiety medications. Preoperative function was assessed using the American Shoulder and Elbow Surgeons (ASES) score, visual analog scale (VAS) for pain, Single Assessment Number Evaluation (SANE), Simple Shoulder Test (SST), and the Veterans RAND 12-Item Health Survey (VR-12). The subjective ASES score measures shoulder comfort and function on a scale of 0 to 100 , with 100 being the highest score. ${ }^{9}$ The VAS pain score was taken from the patient response to the ASES question "How bad is your pain today?." Responses could range from 0 to 10 with 0 being "no pain at all" and 10 being "pain as bad as it could be." The SANE is an outcomes measure in which patients answer the question, "How would you rate your shoulder today as a percentage of being normal $(0 \%$ to $100 \%$ scale with $100 \%$ being normal)?." ${ }^{10}$ The SST is a 12 question survey that measures comfort and physical function of the shoulder. ${ }^{11}$ The VR-12 is a health-related quality of life assessment in which patients answer questions related to eight domains of physical and mental health. A composite score is generated, which can be compared with the mean U.S. population score of $50 .^{12}$

Patients were administered the Hospital for Special Surgery's (HSS) Shoulder Surgery Expectations Survey to assess preoperative expectations. ${ }^{1}$ This instrument is a validated and reproducible patient-reported instrument for assessing the expectations related to symptom relief, physical function, and psychosocial function of patients undergoing shoulder surgery. Patients rate the importance of 17 different expectations of shoulder surgery as "very important," "somewhat important," "a little important," "I do not expect this," or "this does not apply to me." For individual expectations statements, scores ranged from 1 to 5 with 1 being "very important," and 5 being "I do not expect this, or this does not apply to me." For the purpose of this study, higher ratings of importance were defined as greater expectations. Scores were determined for each of the 17 items in the questionnaire in addition to a raw cumulative score $(0-80$ scale) which was then converted [(raw score $/ 80) \times 100$ ] to a 0 to 100 scale with 100 indicating highest expectations. Both cumulative and individual statement expectations scores were analyzed. To limit the number of tests performed, 6 individual expectations statements that represented activities requiring higher level of function were selected for comparison. These statements included "Improve ability to carry objects over 10 pounds," "Improve ability to reach above shoulder level," "Improve ability to exercise or participate in recreational sports," "Improve ability to participate in overhead sports," "Improve ability to participate in recreational activities," and "For the shoulder to be back to the way it was."

All surveys, including the HSS Shoulder Expectations Survey, were administered after the patient was indicated for surgery and underwent the preoperative counseling and informed consent process at the discretion of the surgeon.

At 2 years postoperatively, patients were contacted via phone or email and were administered ASES, SANE, and satisfaction surveys. The satisfaction survey asked patients to respond to the question "How satisfied are you with the 
Table 1 Patient demographics

\begin{tabular}{|c|c|c|c|c|c|c|c|}
\hline Measure & aTSA & Range & SD & RTSA & Range & SD & $p$-Value \\
\hline Number of patients & 64 & - & - & 64 & - & - & - \\
\hline Age $(y)$ & 65.7 & $36.6-86.3$ & 9.6 & 71.4 & $51.1-91.4$ & 8.8 & 0.002 \\
\hline Gender & $22 / 64$ (34.4\%) female & - & - & $41 / 64$ (64.1\%) female & - & - & 0.001 \\
\hline BMI & 30.0 & $19.3-45.9$ & 6.3 & 28.7 & $19.5-46.5$ & 5.3 & 0.360 \\
\hline Number of comorbidities & 3.3 & $0.0-14.0$ & 2.6 & 3.7 & $1.0-9.0$ & 1.8 & 0.087 \\
\hline
\end{tabular}

Abbreviations: aTSA, anatomic total shoulder arthroplasty; BMI, body mass index; RTSA, reverse total shoulder arthroplasty; SD, standard deviation.

result of your shoulder replacement?" with responses "very satisfied," "somewhat satisfied," "neutral," "somewhat dissatisfied," or "very dissatisfied."

There is no established minimal clinically important difference (MCID) in HSS Shoulder Surgery Expectations Survey score; therefore, a power analysis could not be reliably performed. Patients were enrolled from March 2014 until June 2015. Groups were compared using $t$-tests for independent groups for continuous variables (or its nonparametric variant, Mann-Whitney if necessary) and Fisher's exact test for categorical variables. When analyzing cumulative expectations in addition to each of the 17 individual expectations survey statements, $p<0.01$ was considered to be considered statistically significant due to the large number of tests performed. For the remainder of the analyses, $p<0.05$ was considered to be statistically significant. For the HSS Shoulder Surgery Expectations Survey, each item as well as the total score was correlated with patient outcomes using the Spearman's correlation coefficient. Multivariate linear regression was performed to analyze if patient demographic variables were associated with expectations.

\section{Results}

\section{Demographics}

A total of 64 patients in the aTSA group and 64 patients in the RTSA group were included in the study. The mean patient age was significantly higher in the RTSA group $(p=0.002)$, and the RTSA group was comprised of more females $(p=0.001)$.
There were no significant differences in mean BMI and mean number of comorbidities between the aTSA and RTSA groups ( - Table 1). Forty-nine patients (76.6\%) in the aTSA group and 43 patients $(67.2 \%)$ in the RTSA group were married $(p=0.335)$. Ten patients $(15.6 \%)$ in the aTSA group and 12 patients (18.8\%) in the RTSA group self-reported depression as a comorbidity $(p=0.815)$. Thirteen patients in the aTSA group and 37 patients in the RTSA group had a history of previous nonarthroplasty shoulder surgery $(p<0.001)$. Twelve of the 13 (92.3\%) previous shoulder surgeries in the aTSA group and 33 of the $37(89.2 \%)$ previous shoulder surgeries in the RTSA group were ipsilateral $(p<0.001)$. Seven of 128 patients enrolled in the study canceled surgery due to changes in their health or for personal reasons.

\section{Preoperative Functional Outcomes Scores}

Patients in the aTSA group had significantly higher preoperative SST and VR-12 mental component scores compared with patients in the RTSA group ( $p=0.014$ and 0.009 , respectively). Patients in the aTSA group had better preoperative active forward elevation compared with those in the RTSA group (109 vs. 81 degrees; $p=0.001$ ). There were no significant differences in preoperative ASES, VAS pain, SANE, and VR-12 physical component scores between the two groups (-Table 2 ).

\section{Expectations}

There was no significant difference in cumulative preoperative expectations in patients undergoing aTSA compared with those undergoing RTSA (median 76.9 vs. 67.5;

Table 2 Preoperative functional outcomes scores

\begin{tabular}{|l|l|l|l|l|l|l|l|}
\hline Measure & aTSA & Range & SD & RTSA & Range & SD & $p$-Value \\
\hline ASES & 32.7 & $0.0-74.1$ & 18.0 & 34.7 & $6.7-80.0$ & 18.7 & 0.606 \\
\hline VAS pain & 6.6 & $0.0-10.0$ & 2.3 & 6.0 & $0.0-10.0$ & 3.1 & 0.267 \\
\hline SANE & 27.0 & $0.0-91.0$ & 25.1 & 25.9 & $0.0-86.7$ & 22.5 & 0.815 \\
\hline SST & 33.5 & $0.0-83.3$ & 22.0 & 23.4 & $0.0-66.7$ & 16.1 & 0.014 \\
\hline VR-12 M & 56.6 & $21.4-67.9$ & 9.9 & 51.2 & $26.3-64.5$ & 9.4 & 0.009 \\
\hline VR-12 P & 35.9 & $20.8-63.2$ & 8.0 & 36.1 & $16.4-55.0$ & 8.0 & 0.914 \\
\hline AFE & 108.6 & $60.0-160.0$ & 28.0 & 80.9 & $0.0-170.0$ & 46.9 & 0.001 \\
\hline
\end{tabular}

Abbreviations: AFE, active forward elevation (degrees); ASES, American Shoulder and Elbow Surgeons score; aTSA, anatomic total shoulder arthroplasty; RTSA, reverse total shoulder arthroplasty; SANE, Single Assessment Number Evaluation; SD, standard deviation; SST, Simple Shoulder Test; VAS, visual analogue scale for pain; VR-12 M, Veterans RAND 12 Item Health Survey Mental Component; VR-12 P, Veterans RAND 12 Item Health Survey Physical Component. 
Table 3 Multivariate linear regression for expectations for improved ability to exercise

\begin{tabular}{|c|c|c|c|c|}
\hline Variable & Unstandardized coefficient & $p$-Value & 95\% Confide & terval \\
\hline & & & Lower limit & Upper limit \\
\hline aTSA & -0.64 & 0.02 & -1.20 & -0.86 \\
\hline Older age & 0.04 & 0.02 & 0.01 & 0.07 \\
\hline Male & 0.23 & 0.42 & -0.33 & 0.77 \\
\hline Married & 0.44 & 0.88 & -0.55 & 0.64 \\
\hline \# Comorbidities & 0.01 & 0.89 & -0.11 & 0.12 \\
\hline Previous failed ipsilateral surgery & -0.19 & 0.56 & -0.84 & 0.46 \\
\hline
\end{tabular}

Abbreviation: aTSA, anatomic total shoulder arthroplasty.

Note: Positive correlations reflect higher score for individual expectation statements, which patients rated 1 to 5 with 1 being "Very important to me" and 5 being "I do not expect this or this does not apply to me," meaning positive correlations reflect lower expectations.

interquartile range $[\mathrm{IQR}] 21.25 ; 23.13 ; Z=-2.19 ; p=0.028$, respectively). Patients in the aTSA group had higher expectations for improved ability to exercise compared with those in the RTSA group (median 2.0 vs. 2.0 ; IQR 1.0, 3.0; $Z=-2.76$; $p=0.005$, respectively). There was no statistically significant difference in expectations on any of the other analyzed items on the HSS Shoulder Surgery Expectations Survey. On univariate analysis, gender, number of comorbidities, and previous failed ipsilateral surgery did not correlate with preoperative expectations in the aTSA and RTSA groups $(p>0.05)$. On multivariate linear regression analysis, aTSA $(p=0.024)$ and younger age $(p=0.018)$ were associated with higher expectations for improved ability to exercise (-Table 3).

\section{Postoperative Functional Outcomes Scores}

Ninety-one of 121 patients (75\%) who underwent surgery were reached for follow-up at a minimum of 2 years postoperatively. Patients who underwent aTSA had higher ASES and SANE scores postoperatively $(p<0.001, p=0.002$, respectively), and also had higher changes in ASES and SANE scores ( $p=0.004, p=0.001$, respectively) compared with those in the RTSA group. There were no significant differences in VAS pain scores or postoperative satisfaction scores between the two groups ( $\mathbf{- T a b l e s} \mathbf{4}$ and $\mathbf{5}$ ).

\section{Relationship between Expectations and Functional Outcomes}

Cumulative preoperative expectations score was not correlated with postoperative functional outcomes score in the aTSA group, RTSA group, or groups combined. In the aTSA group, preoperative expectations for ability to exercise were positively correlated with change in preoperative to postoperative ASES $(R=0.362 ; p=0.01)$ and SANE scores $(R=0.360 ; p=0.01)$. Other individual preoperative expectation statements were not correlated with functional outcomes in the aTSA, RTSA, or combined groups. Preoperative expectations scores, including cumulative score and individual expectations statements, were not correlated with postoperative satisfaction scores in the aTSA, RTSA, or combined groups.

\section{Discussion}

The results of this study indicate that patients undergoing aTSA and RTSA have similar cumulative preoperative expectations; however, patients undergoing aTSA appear to have greater expectations for higher-level activities, such as participating in exercise. Additionally, we found that older patients undergoing RTSA had lower expectations for ability to participate in exercise. In line with previous literature, we

Table 4 Postoperative functional outcomes scores

\begin{tabular}{|l|l|l|l|l|l|l|}
\hline Measure & aTSA & Interquartile range & RTSA & Interquartile range & $p$-Value & $Z$ \\
\hline ASES & 95.0 & 15.0 & 83.3 & 20.0 & $<0.001$ & -3.97 \\
\hline VAS pain & 0.0 & 2.0 & 1.0 & 2.5 & 0.251 & -0.73 \\
\hline SANE & 90.0 & 11.8 & 83.0 & 33.0 & 0.002 & -3.13 \\
\hline Delta ASES & 55.3 & - & 43.2 & - & 0.004 & - \\
\hline Delta VAS & -5.4 & - & -4.4 & - & 0.141 & - \\
\hline Delta SANE & 60.5 & - & 46.6 & - & 0.001 & - \\
\hline Satisfaction & 1.0 & 0.0 & 1.0 & 1.0 & 0.162 & -1.37 \\
\hline
\end{tabular}

Abbreviations: ASES: American Shoulder and Elbow Surgeons score; aTSA, anatomic total shoulder arthroplasty; RTSA, reverse total shoulder arthroplasty; SANE, Single Assessment Number Evaluation; VAS, visual analog scale for pain.

Note: Patient-reported satisfaction scored $1-5$ with 1 being "very satisfied" and 5 being "very dissatisfied." ASES, VAS pain, SANE, and satisfaction reported as median values. Delta ASES, delta VAS, and delta SANE reported as mean values. 
Table 5 Postoperative satisfaction scores

\begin{tabular}{|l|l|l|l|l|l|}
\hline & Very satisfied & Somewhat satisfied & Neutral & Somewhat dissatisfied & Very dissatisfied \\
\hline aTSA & $41 / 50(82 \%)$ & $6 / 50(12 \%)$ & $1 / 50(2 \%)$ & $2 / 50(4 \%)$ & $0 / 50(0 \%)$ \\
\hline RTSA & $29 / 41(70.7 \%)$ & $6 / 41(14.6 \%)$ & $2 / 41(4.9 \%)$ & $2 / 41(4.9 \%)$ & $2 / 41(4.9 \%)$ \\
\hline Total & $70 / 91(76.9 \%)$ & $12 / 91(13.2 \%)$ & $3 / 91(3.3 \%)$ & $4 / 91(4.4 \%)$ & $2 / 91(2.2 \%)$ \\
\hline
\end{tabular}

Abbreviations: aTSA, anatomic total shoulder arthroplasty; RTSA, reverse total shoulder arthroplasty.

report that patients who underwent aTSA had higher postoperative functional outcomes than those who underwent RTSA $^{5,7}$ In a comparison of postoperative outcomes, Flurin et $\mathrm{al}^{5}$ showed that patients who underwent RTSA had significantly lower SST scores and range of motion compared with patients who underwent aTSA. Puskas et $\mathrm{al}^{7}$ further corroborated these findings by demonstrating greater preoperative and postoperative impairment and lower strength and range of motion in patients undergoing RTSA compared with aTSA.

Previous literature has analyzed expectations of shoulder arthroplasty and associations with postoperative outcomes. 2,4,8,13,14 Henn et $\mathrm{al}^{4}$ found younger patients had greater expectations of aTSA; however, previous studies ${ }^{13,14}$ have not found an association between patient age and expectations of RTSA. Our investigation demonstrated younger patients had higher expectations for improved ability to exercise. Previous study also reported patients with glenohumeral osteoarthritis, higher preoperative function, and no history of joint replacement had greater expectations of RTSA. Similar to our findings, Rauck et $\mathrm{al}^{13}$ demonstrated cumulative preoperative expectations of RTSA were not associated with improved outcomes; however, particular expectations, such as relief of nighttime pain and ability to participate in nonoverhead sports, were associated with improved functional outcomes. Our investigation demonstrated that there is some association between preoperative expectations and postoperative functional outcome. Although cumulative preoperative expectations score was not correlated with postoperative functional outcome, higher expectations for the ability to exercise were associated with increased change in preoperative to postoperative functional scores in the aTSA group. In 2016, one study ${ }^{15}$ reported that men more often expect to return to sports following shoulder arthroplasty while women tend to expect to be able to return to household chores, and both genders had high levels of achieving their expectations. Another study ${ }^{16}$ reported an association between self-reported motivation to return to sports and postoperative return to sports following total hip arthroplasty. Patients who desire to return to higher functioning levels of activity such as exercise may be more motivated with the postoperative rehabilitation process.

Despite aTSA yielding better functional outcomes than RTSA in this study and other studies, ${ }^{5,7}$ patients in both groups had the similar aggregate preoperative expectations and postoperative satisfaction scores. Qualitative research demonstrates expectations for recovery after orthopaedic injury are based on physician diagnosis and treatment, prior experiences with injury, others' experiences and attitudes, information from the Internet, and a sense of self-resilience. ${ }^{17}$ Literature shows patient satisfaction is associated with met expectations, particularly regarding information and explanation of medical condition and treatment. ${ }^{18,19}$ Patients in both the aTSA and RTSA groups had high rates of postoperative satisfaction, indicating their expectations were met. Our findings that RTSA patients have lower preoperative functional scores and lower expectations for high-level activities may indicate that patients' expectations of surgery were appropriate, and they received satisfaction more from pain relief rather than ability to participate in higher level of functional activities.

This study has several limitations. The sample size is relatively small and given the lack of an MCID for the expectations scale used, we are unable to calculate one. As a result, it is possible that nonsignificant findings are a result of inadequate power. Furthermore, while the consent forms and educational materials were consistent for all surgeons, patients may have had variable levels of preoperative education from other sources (family, friends, Internet, etc.). This lack of consistency could have influenced the expectations, but we believe that this is most reflective of the true clinical situation. Given that patients were included from the practices of five surgeons, the sample size did not allow comparison of expectations between surgeons. Prior study has shown that current mechanisms of preoperative counseling did not influence patients' preoperative expectations of shoulder surgery. ${ }^{20}$

\section{Conclusion}

Though patients undergoing primary aTSA demonstrated greater improvement in functional outcome than those undergoing primary RTSA, both groups reported the same aggregate preoperative expectations. Those undergoing aTSA and younger patients have higher expectations for higher level activities, including exercise. In the aTSA group, patients' preoperative expectations for return to exercise were positively correlated with postoperative functional outcomes.

Note

Investigation was performed at the Rothman Institute, Thomas Jefferson University Hospitals, Philadelphia, Pennsylvania.

\section{Conflict of Interest}

M.L. reports personal fees from Tornier, outside the submitted work; In addition, M.L. has a patent Shoulder 
Arthroplasty with royalties paid to Tornier. G.W. reports other from Pennsylvania Orthopaedic Society, other from American Shoulder and Elbow Surgeons, other from Operative Techniques in Orthopaedic Surgery, other from Techniques in Shoulder and Elbow Surgery, other from American Academy of Orthopaedic Surgeons, grants and personal fees from DePuy, personal fees from DJO, personal fees from DePuy, Mitek, other from In vivo Therapeutics, outside the submitted work; In addition, G.W. has a patent Shoulder Arthroplasty licensed to DePuy, a patent Shoulder Arthroplasty issued to IMDS/ Cleveland Clinic, a patent Shoulder Arthroplasty with royalties paid to DJO, and a patent Shoulder Arthroplasty with royalties paid to Lippincott. S.N. reports other from Arthrex Inc, other from DJO Surgical, other from Flexion Therapeutics, other from Force Therapeutics, other from Integra LifeSciences, other from Miami Device Solutions, other from Parvizi Surgical Solutions, other from Synthes, other from ZimmerBiomet, other from Saunders Mosby-Elsevier, other from RubiconMD, other from MD Live, other from MD Valuate, outside the submitted work. J.A. reports other from DJO, other from Integra Life Sciences, other from Tornier, other from DePuy Synthes, other from Lippincott, Williams \& Wilkins, outside the submitted work.

\section{References}

1 Mancuso CA, Altchek DW, Craig EV, et al. Patients' expectations of shoulder surgery. J Shoulder Elbow Surg 2002;11(06):541-549

2 Henn RF III, Kang L, Tashjian RZ, Green A. Patients' preoperative expectations predict the outcome of rotator cuff repair. J Bone Joint Surg Am 2007;89(09):1913-1919

3 Mahomed NN, Liang MH, Cook EF, et al. The importance of patient expectations in predicting functional outcomes after total joint arthroplasty. J Rheumatol 2002;29(06):1273-1279

4 Henn RF III, Ghomrawi H, Rutledge JR, Mazumdar M, Mancuso CA, Marx RG. Preoperative patient expectations of total shoulder arthroplasty. J Bone Joint Surg Am 2011;93(22):2110-2115

5 Flurin PH, Marczuk Y, Janout M, Wright TW, Zuckerman J, Roche CP. Comparison of outcomes using anatomic and reverse total shoulder arthroplasty. Bull Hosp Jt Dis (2013) 2013;71 (Suppl 2):101-107

6 Ponce BA, Oladeji LO, Rogers ME, Menendez ME. Comparative analysis of anatomic and reverse total shoulder arthroplasty: in-hospital outcomes and costs. J Shoulder Elbow Surg 2015;24(03):460-467

7 Puskas B, Harreld K, Clark R, Downes K, Virani NA, Frankle M. Isometric strength, range of motion, and impairment before and after total and reverse shoulder arthroplasty. J Shoulder Elbow Surg 2013;22(07):869-876

8 Swarup I, Henn CM, Nguyen JT, et al. Effect of pre-operative expectations on the outcomes following total shoulder arthroplasty. Bone Joint J 2017;99-B(09):1190-1196

9 Richards RR, An KN, Bigliani LU, et al. A standardized method for the assessment of shoulder function. J Shoulder Elbow Surg 1994; 3(06):347-352

10 Williams GN, Gangel TJ, Arciero RA, Uhorchak JM, Taylor DC. Comparison of the Single Assessment Numeric Evaluation method and two shoulder rating scales. Outcomes measures after shoulder surgery. Am J Sports Med 1999;27(02):214-221

11 Lippit SB, Harryman DT, Matsen FAI. A practical tool for evaluating function: the Simple Shoulder Test. In: Matsen FAI, Fu FH, Hawkins RJ, eds. The Shoulder: A Balance of Mobility and Stability. Rosemont, IL: American Academy of Orthopaedic Surgeons; 1993:501-518

12 Selim AJ, Rogers W, Fleishman JA, et al. Updated U.S. population standard for the Veterans RAND 12-item Health Survey (VR-12). Qual Life Res 2009;18(01):43-52

13 Rauck RC, Swarup I, Chang B, et al. Effect of preoperative patient expectations on outcomes after reverse total shoulder arthroplasty. J Shoulder Elbow Surg 2018;27(11):e323-e329

14 Rauck RC, Swarup I, Chang B, et al. Preoperative patient expectations of elective reverse shoulder arthroplasty. J Shoulder Elbow Surg 2019;28(07):1217-1222

15 Jawa A, Dasti U, Brown A, Grannatt K, Miller S. Gender differences in expectations and outcomes for total shoulder arthroplasty: a prospective cohort study. J Shoulder Elbow Surg 2016;25(08): 1323-1327

16 Ollivier M, Frey S, Parratte S, Flecher X, Argenson JN. Pre-operative function, motivation and duration of symptoms predict sporting participation after total hip replacement. Bone Joint J 2014;96-B (08):1041-1046

17 Carroll LJ, Lis A, Weiser S, Torti J. How well do you expect to recover, and what does recovery mean, anyway? Qualitative study of expectations after a musculoskeletal injury. Phys Ther 2016;96 (06):797-807

18 Bible JE, Shau DN, Kay HF, Cheng JS, Aaronson OS, Devin CJ. Are low patient satisfaction scores always due to the provider? Determinants of patient satisfaction scores during spine clinic visits. Spine 2018;43(01):58-64

19 Hageman MG, Briët JP, Bossen JK, Blok RD, Ring DC, Vranceanu AM. Do previsit expectations correlate with satisfaction of new patients presenting for evaluation with an orthopaedic surgical practice? Clin Orthop Relat Res 2015;473(02): 716-721

20 Lawrence C, Zmistowski BM, Lazarus M, Abboud J, Williams G, Namdari S. Expectations of shoulder surgery are not altered by surgeon counseling of the patient. Joints 2017;5(03): 133-137 